\title{
Metformin induces weight loss associated with gut microbiota alteration in non-diabetic obese women: a randomized double-blind clinical trial
}

\author{
Hanieh-Sadat Ejtahed' ${ }^{1}$, Raul Y Tito², Seyed-Davar Siadat ${ }^{3}$, Shirin Hasani-Ranjbar', Zahra Hoseini-Tavassol ${ }^{3}$, \\ Leen Rymenans ${ }^{2}$, Kristin Verbeke ${ }^{4}$, Ahmad-Reza Soroush ${ }^{1, *}$, Jeroen Raes ${ }^{2, *}$ and Bagher Larijani ${ }^{5}$
}

${ }^{1}$ Obesity and Eating Habits Research Center, Endocrinology and Metabolism Clinical Sciences Institute, Tehran University of Medical Sciences, Tehran, Iran, ${ }^{2}$ Department of Microbiology and Immunology, Rega Institute, KU Leuven, Leuven, Belgium, ${ }^{3}$ Department of Mycobacteriology and Pulmonary Research, Microbiology Research Center, Pasteur Institute of Iran, Tehran, Iran, ${ }^{4}$ Translational Research in Gastrointestinal Disorders, KU Leuven, Leuven, Belgium, and ${ }^{5}$ Endocrinology and Metabolism Research Center, Endocrinology and Metabolism Clinical Sciences Institute, Tehran University of Medical Sciences, Tehran, Iran

Correspondence should be addressed to A-R Soroush or J Raes Email soroush.journal@gmail.com or jeroen.raes@med. kuleuven.be

\begin{abstract}
Objective: The increasing prevalence of obesity over the past few decades constitutes a global health challenge. Pharmacological therapy is recommended to accompany life-style modification for obesity management. Here, we perform a clinical trial to investigate the effects of metformin on anthropometric indices and gut microbiota composition in non-diabetic, treatment-naive obese women with a low-calorie diet (LCD).

Design: Randomized double-blind parallel-group clinical trial

Methods: Forty-six obese women were randomly assigned to the metformin (500 mg/tab) or placebo groups using computer-generated random numbers. Subjects in both groups took two tablets per day for 2 months. Anthropometric measurements and collection of blood and fecal samples were done at the baseline and at the end of the trial. Gut microbiota composition was assessed using $16 \mathrm{~S}$ rRNA amplicon sequencing. Results: Twenty-four and twenty-two subjects were included in the metformin + LCD and placebo + LCD groups, respectively; at the end of trial, 20 and 16 subjects were analyzed. The metformin + LCD and placebo + LCD caused a 4.5 and $2.6 \%$ decrease in BMI from the baseline values, respectively $(P<0.01)$. Insulin concentration decreased in the metformin $+\mathrm{LCD}$ group $(P=0.046)$. The overall fecal microbiota composition and diversity were unaffected in the metformin + LCD group. However, a significant specific increase in Escherichia/Shigella abundance was observed after metformin + LCD intervention $(P=0.026)$. Fecal acetate concentration, but not producers, was significantly higher in the placebo $+\mathrm{LCD}$ group, adjusted for baseline values and BMI $(P=0.002)$.

Conclusions: Despite the weight reduction after metformin intake, the overall fecal microbiota composition remained largely unchanged in obese women, with exception of changes in specific proteobacterial groups.
\end{abstract}




\section{Introduction}

The obesity epidemic is an increasing public health and financial burden across the world $(1,2)$. It is estimated that the prevalence of obesity increased $33 \%$ by 2030 (3). Obesity is often associated with insulin resistance, making obese individuals susceptible to metabolic syndrome and its related cardiometabolic disorders (4). Because obesity management with life-style modification is an important challenge for most individuals, adjunct treatments like pharmacological therapy are frequently applied (5). Given the modest yet durable weight loss observed following metformin consumption in diabetics, women suffering from polycystic ovary syndrome and healthy obese adults, its administration in combination with lifestyle modification might be helpful for combating obesity (6), although further investigations are needed to confirm its efficacy in nondiabetic obese individuals.

Metformin, the first-line drug for treating type 2 diabetes, mediates its glucose-lowering property and its welcome weight-reducing side effect by inhibition of liver glucose production, appetite suppression, improvement of insulin sensitivity and regulation of fat oxidation and storage (7). Moreover, recent evidence suggests that gut microbiota alterations may contribute to these effects (8, 9). Composition of the gut microbiota, a complex and dynamic population of microorganisms living in the intestine, is shaped by several environmental factors such as diet and drugs (10).

Previous studies investigated the effect of metformin on gut microbiota composition in diabetic subjects (11, $12,13,14,15)$. However, considering that the effect of metformin on gut microbiota composition differs under healthy and diabetes conditions $(16,17)$, it should be assessed in healthy obese subjects too. Obesity is accompanied by an altered gut microbiota, suggested to be linked to increased energy harvest $(18,19,20$, 21). Gut bacteria-released metabolites have the effective roles in weight control by stimulating gut satietogenic hormones, controlling lipid metabolism in adipose tissue, influencing insulin signaling and improving gut barrier function (20).

To assess gut microbial mediation of weight loss effects of metformin, we conducted a randomized double-blind parallel-group clinical trial investigating the effects of metformin on anthropometric indices and gut microbiota composition in obese women with a lowcalorie diet (LCD).

\section{Subjects and methods}

\section{Subjects}

This study was a single site, randomized, double-blind, placebo-controlled parallel group clinical trial, which was conducted at the Obesity Clinic of Shariati Hospital in Tehran, Iran from October 2017 to March 2018. Subjects were recruited via advertising at the clinic and Shariati Hospital website and social media channels. Participants were eligible for the study if they met the following inclusion criteria: women aged between 20 and 45 years, BMI in the range of $30-40 \mathrm{~kg} / \mathrm{m}^{2}$, willingness to adhere to the study protocol. Exclusion criteria were pregnancy and lactation, smoking, having cardiovascular disease, kidney and liver disorders, inflammatory bowel diseases, diabetes and cancer, history of digestive tract surgery, history of acute and chronic diarrhea over the last month before the start of the study, antibiotic therapy within the 2 months prior to or during the study period, multivitamin supplementation during the study, routine use of probiotics and prebiotics products over the last month before the start of the study, routine use of antiinflammatory drugs within 1 month prior to the start of the study, taking weight loss drugs over the last 3 months, history of specific weight loss diet over the last 3 months and history of mental illness.

Sample size was determined for BMI. For an expected change of $2 \mathrm{~kg} / \mathrm{m}^{2}$ between metformin and placebo groups and by considering an alpha value equal to 0.05 and a power of $80 \%$, the sample size was computed as 16 subjects in each group. Considering a $20 \%$ drop-out rate, the sample size has been increased up to 20 subjects per group.

The written informed consent was obtained from all participants. All procedures involving human subjects were approved by ethical committee of Endocrinology and Metabolism Research Institute of Tehran University of Medical Sciences (ID number: IR.TUMS.EMRI. REC.1395.0090) and the trial was registered in the Iranian Registry of Clinical Trials (IRCT) with code of IRCT20090420001825N2.

\section{Study design}

In this study, 46 subjects were randomly assigned to the metformin $(n=24)$ or placebo $(n=22)$ group by a balanced block randomization procedure using computer-generated random numbers. For a period of 2 months, patients had 
daily intake of two tablets of metformin (each tablet: $500 \mathrm{mg}$, Gly-once, Koushanpharmed Co., Iran) or placebo (containing lactose and starch) with main meals. Verbal and written instructions on how to take the tablets were provided at the initial visit. Besides, both groups were instructed to maintain a reduction in daily caloric intake of $500 \mathrm{kcal}$ during the study. The composition of the prescribed diet was 55\% carbohydrate, 30\% fat and 15\% protein. Subjects in both groups of the study received physical activity advice, encouraging physical activity by walking fast for $30 \mathrm{~min}$ a day. Necessary recommendations regarding not changing the medications and avoiding other supplement intake during the study period were given to the participants.

Both participants and investigators were blinded to the treatment allocation. The metformin and placebo tablets were packed identically and separated by code. To implement the allocation concealment, sequentially numbered, opaque sealed containers with identical appearance were used. Participants were asked to bring the remaining tablets at the end of study for assessing the compliance. Participants were defined as non-compliant if they had taken less than $80 \%$ of the tablets. Adverse events and compliance were monitored for each participant by investigators during weekly phone calls.

\section{Anthropometric, dietary and biochemical measurements}

Demographic questionnaire was completed for each participant at the screening visit and medical history, prescribed and non-prescribed medications and dietary intakes were recorded. Anthropometric measurements were done at baseline and after 2 months. Height was measured without shoes using a stadiometer with a precision of $0.5 \mathrm{~cm}$. Participants were weighed with light clothes without shoes using a digital scale (Seca, Germany) with an accuracy of $0.1 \mathrm{~kg}$. BMI was calculated as body weight $(\mathrm{kg})$ divided by the square of the height $\left(\mathrm{m}^{2}\right)$. Waist circumference was measured at the midpoint between the last rib and the iliac crest and hip circumference was measured at the widest portion of the buttocks with a precision of $0.1 \mathrm{~cm}$. Waist-to-hip ratio (WHR) is calculated as waist measurement divided by hip measurement and waist to height ratio (WHtR) is the ratio of the circumference of the waist to the height measurement. Assessment of body composition was done using Dual-energy X-ray absorptiometry method (DEXA) by Lunar DPX-MD device (Lunar Corporation, USA).
The validated 147-item semi-quantitative food frequency questionnaire (FFQ) was completed at baseline to assess energy and macronutrients intakes. The frequency of food intake over the past year was interviewed by a trained nutritionist. The reliability and validity of the FFQ have been evaluated as acceptable (22, 23). Due to the incompletion and limitations of Iranian Food Composition Table, the Food Composition Database of United States Department of Agriculture (USDA) has been used to analyze foods and beverages.

Blood samples were taken after $12-14 \mathrm{~h}$ of overnight fasting before and after the intervention. Serum was immediately separated by centrifuging samples at $1300 \boldsymbol{g}$ for $10 \mathrm{~min}$ at the room temperature and stored at $-80^{\circ} \mathrm{C}$ freezer until analysis. Serum glucose, total cholesterol (TC), triglyceride (TG), high-density lipoprotein cholesterol (HDL-C), low-density lipoprotein cholesterol (LDL-C), alanine aminotransferase (ALT), aspartate aminotransferase (AST) and high-sensitivity C-reactive protein (hsCRP) concentrations were measured by Roche kits using auto-analyzer instrument (Hitachi, Cobas C 311, Roche Diagnostics $\mathrm{GmbH}$ ). Serum insulin concentration was measured by an enzyme immunoassay kit (Monobind Inc., Lake Forest, CA, USA). Insulin resistance index was calculated by the homeostasis model assessment of insulin resistance (HOMA-IR) equation: HOMA-IR $=[\mathrm{FBS}$ $(\mathrm{mg} / \mathrm{dL}) \times$ fasting inulin concentration $(\mathrm{mU} / \mathrm{L}) / 405]$. Lipoprotein lipase (LPL), glucagon-like peptide 1 (GLP-1) (ZellBio GmbH, Germany) and fasting-induced adipose factor (FIAF) (BioVendor, Germany) were determined by ELISA kits.

\section{Fecal water content and microbiota profiling}

Stool samples were collected at baseline and 2 months after intervention using a stool specimen collection kit, brought to the clinic in ice packs. Samples were stored at $-80^{\circ} \mathrm{C}$ for fecal microbial and metabolite profiling. Fecal water content was measured by weighing frozen stool samples before and after lyophilization (Christ Alpha 1-4 LSCbasic Freeze Dryer, Germany). The analyses of gut microbiota taxonomic composition, fecal DNA extraction, library preparation and 16S rRNA gene sequencing were performed as described in Tito et al. (24). In brief, we used PowerMicrobiome RNA Isolation Kit (MOBIO Laboratories Inc., Germany) for DNA extraction and sequencing was done using the Illumina MiSeq platform at Nucleomics core, KU Leuven. 16S data pre-processing was performed using LotuS (25) and DADA2 (26) pipelines and taxonomy assignment with 
the RDP classifier v 2.12 (27), with default parameters. After removing reads annotated to the class Chloroplast, family mitochondria and unclassified bacteria, data were rarefied to 10000 reads per sample, alpha-diversity and beta-diversity indices were generated using the vegan and phyloseq $\mathrm{R}$ packages. Richness and Simpson indices were used in microbial alpha-diversity measurements based on operational taxonomic units (OTUs). Richness is the number of different kinds of microorganisms present in a particular community and Simpson takes into account the relative abundance, as well as the Richness. All further analyses were performed at genus level. Enterotyping (or community typing) using the DMM approach was performed in $\mathrm{R}$ as described previously (28). To increase accuracy, enterotyping was performed on a combined genus-abundance matrix that included study and disease cohort samples, complemented with 1106 samples from the FGFP (29). Microbiome variation among individuals was visualized by PCoA using Bray-Curtis dissimilarity on the genus-abundance matrix.

\section{Targeted metabolomics analysis of fecal samples}

Fecal short-chain fatty acids (SCFAs) were measured using gas chromatography. Approximately $100 \mathrm{mg}$ of fecal sample was suspended in $1 \mathrm{~mL}$ of saturated $\mathrm{NaCl}$ (36\%) solution. An internal standard $(50 \mu \mathrm{L}$ of $10.7 \mu \mathrm{M} 2$-ethylbutyric acid by Merck (München, Germany) in MQ water) was added and the samples were homogenized with glass beads. After adding $150 \mu \mathrm{L}$ of $96 \% \mathrm{H}_{2} \mathrm{SO}_{4}$, SCFAs were extracted in $3 \mathrm{~mL}$ of ether. The ether layer was collected and dried with $\mathrm{Na}_{2} \mathrm{SO}_{4}(150 \mathrm{mg})$. The supernatant $(0.5 \mu \mathrm{L})$ was analyzed by a gas chromatography-flame ionization detection (GCFID) method (Agilent). The system was equipped with a DB FFAP analytical column $(30 \mathrm{~m} \times 0.53 \mathrm{~mm}$ ID, $1.0 \mu \mathrm{m}$; Agilent) and helium GC grade (5.6) was used as carrier gas with a constant flow of $4.2 \mathrm{~mL} / \mathrm{min}$. The initial oven temperature was held at $100^{\circ} \mathrm{C}$ for $3 \mathrm{~min}$, ramped with $4^{\circ} \mathrm{C} / \mathrm{min}$ to $140^{\circ} \mathrm{C}$ (isothermal for $5 \mathrm{~min}$ ) and further with $40^{\circ} \mathrm{C} / \mathrm{min}$ to $235^{\circ} \mathrm{C}$ (isothermal for $15 \mathrm{~min}$ ). The resulting chromatograms were processed using ChemStation (Agilent Technologies). Acetate, propionate and butyrate were quantified with appropriate calibration curves obtained from internal standard quantitation.

\section{Statistical analysis}

All statistical analyses were performed using R version 3.5.0. A $P$ value $<0.05$ was defined as the level of significance.
The primary outcome was change in BMI during the 8 weeks of the trial. Secondary outcomes included waist and hip circumferences, WHR, WHtR, serum FBS, insulin, lipid profile, ALT, AST, GLP-1, hsCRP, FIAF and LPL concentrations, HOMA-IR, faecal SCFAs and gut microbiota composition. Data were expressed as mean \pm standard deviation or frequency (\%) for quantitative and qualitative variables. The normality distribution for variables was tested by the Kolmogorov-Smirnov test. For insulin, HOMA, TG, GLP1, LPL, AST, ALT and hsCRP, which did not follow normal distribution, analyses were performed after log transformation. Chi-square test was used to determine associations between categorical variables. Comparison of quantitative baseline variables between two groups was done using independent-samples $t$ tests. The changes in biochemical variables and anthropometric measurements of the subjects between the beginning and end of the trial were compared by a paired-sample $t$ test. Primary and secondary outcomes were compared between two groups using generalized linear models adjusting for baseline measurements and BMI. For microbiota-related variables with non-normal distribution, Wilcoxon ranksum or Wilcoxon signed-rank tests were used to compare two independent groups or repeated observations of the same group, respectively. Differences in alpha diversity indices and relative abundance of taxa within each group were determined using the Wilcoxon signed-rank test and the DESeq package. The association between biochemical and anthropometric variables and gut microbial diversity indices were determined using the Spearman correlation test. The Benjamini-Hochberg false discovery rate adjustment was used for multiple testing (30).

\section{Results}

\section{Study participants}

Twenty-four and 22 obese women were included in the metformin +LCD and placebo +LCD groups respectively. Twenty women in the metformin group and 16 women in the placebo group completed the 2-month trial and were included in the statistical analysis. In the metformin group, one participant was lost to follow-up. A total of eight participants, three from the metformin group and five from the placebo group, dropped out of the study, stating loss of interests as the reason for discontinuation. Moreover, one person in the placebo group was excluded from analysis because of lack of procured stool samples 


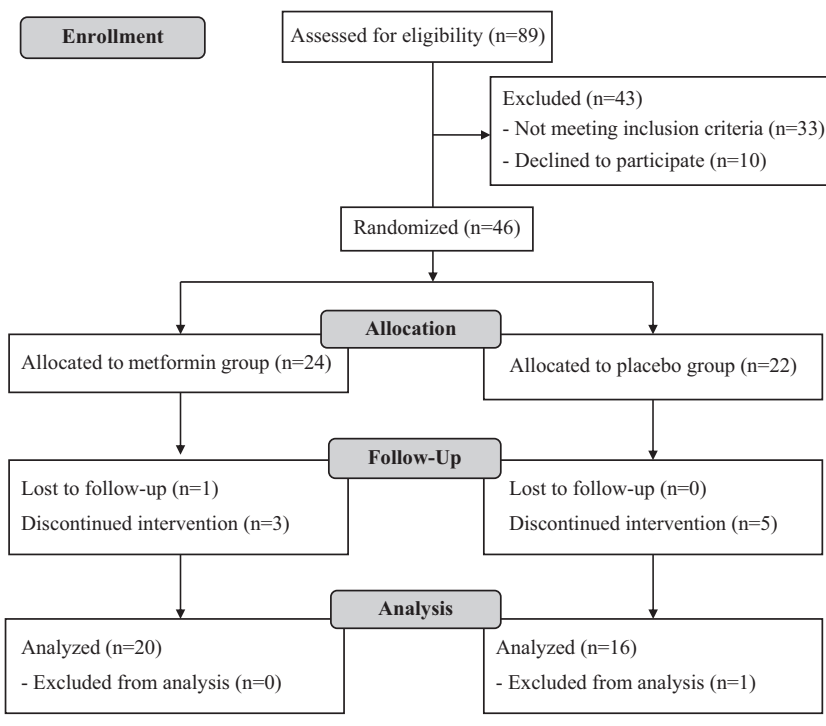

\section{Figure 1}

Flow diagram of study participants.

(Fig. 1). The completion rate of study was slightly higher in the metformin group ( $83 \%$ vs $73 \%$ ). According to tablet counting, the compliance to treatments in both groups was above $80 \%$.

The baseline characteristics of the participants in the two groups are shown in Table 1 . The participants' mean age in the metformin and placebo groups was 37.4 and 34.2 years, respectively $(P=0.17)$. The baseline BMI, hip circumference and WHtR in the metformin group were significantly higher than of those in the placebo group $(P<0.05)$. No statistically significant differences in other anthropometric indices, body composition, bone mineral density (BMD) and dietary intakes were observed between the study groups at the beginning of the study.

The general rate of side effects from the interventions was $11 \%$. Two patients in the intervention group ( 2 out of $20,10 \%$ ) complained about fatigue, flatulence and diarrhea; two in the placebo group ( 2 out of $16,12.5 \%$ ) reported flatulence and sleeplessness. The proportion of participants experiencing any adverse events was comparable between groups.

\section{Effect of intervention on anthropometric indices}

Both the metformin and control groups showed a decrease in BMI from the baseline values, 4.5 and $2.6 \%$, respectively $(P<0.01)$. The BMI reduction was higher in the metformin group than that in the placebo group, adjusted for baseline BMI $(P=0.016)$. Weight, waist and
Table 1 Baseline characteristics and dietary intakes of the study participants. Data are presented as mean \pm S.D.

Comparisons were made with independent samples $t$ test.

\begin{tabular}{|c|c|c|c|}
\hline Variables & $\begin{array}{l}\text { Metformin } \\
\quad(n=20)\end{array}$ & $\begin{array}{c}\text { Placebo } \\
(n=16)\end{array}$ & $P$ value \\
\hline Age (years) & $37.4 \pm 6.97$ & $34.24 \pm 6.82$ & 0.17 \\
\hline \multicolumn{4}{|l|}{ Body composition } \\
\hline BMI $\left(\mathrm{kg} / \mathrm{m}^{2}\right)$ & $35.0 \pm 3.4$ & $32.7 \pm 2.2$ & 0.02 \\
\hline Lean body mass (kg) & $45.8 \pm 7.1$ & $44.1 \pm 3.1$ & 0.45 \\
\hline Fat mass (kg) & $41.4 \pm 10.2$ & $38.1 \pm 5.4$ & 0.32 \\
\hline Body fat percentage (\%) & $45.9 \pm 3.3$ & $44.9 \pm 3.9$ & 0.46 \\
\hline $\mathrm{BMD}\left(\mathrm{g} / \mathrm{cm}^{2}\right)$ & $1.15 \pm 0.06$ & $1.18 \pm 0.07$ & 0.28 \\
\hline \multicolumn{4}{|l|}{ Dietary intake } \\
\hline Energy (kcal/day) & $2955 \pm 836.5$ & $2614 \pm 657.5$ & 0.25 \\
\hline Carbohydrate (g/day) & $465.3 \pm 140.3$ & $397.9 \pm 117.6$ & 0.19 \\
\hline Protein (g/day) & $98.9 \pm 33.5$ & $93.9 \pm 23.8$ & 0.66 \\
\hline Fat (g/day) & $91.2 \pm 31.1$ & $83.3 \pm 23.7$ & 0.46 \\
\hline Fiber (g/1000 kcal) & $19.1 \pm 4.3$ & $19.8 \pm 7.2$ & 0.72 \\
\hline
\end{tabular}

$\mathrm{BMI}$, body mass index; BMD, bone mineral density; WHR, waist-to-hip ratio; WHtR, waist to height ratio.

hip circumferences decreased statistically significant in both groups and there were no significant differences between two groups after adjusting for baseline values. The WHR reduction was significant only in the metformin group $(P=0.033)$. However, the WHtR was significantly decreased in both groups during the study $(P<0.001$ for both groups) (Table 2).

\section{Effect of intervention on serum biochemical variables}

Concentrations of biochemical variables before and after the intervention in obese women are shown in Table 2. Compared to the baseline value, fasting blood sugar (FBS) was significantly increased in the placebo group after the intervention $(P=0.019)$. Insulin concentration was decreased in both groups during the study, although this reduction was significant only in the metformin group $(P=0.046)$. HOMA-IR had a decreasing trend in both groups; however, it was statistically non-significant. TC and LDL-C were significantly decreased in comparison to the baseline values in the placebo group $(P<0.05)$, and their concentrations were different between the two groups after adjusting for baseline values and BMI $(P<0.05)$. In contrast, HDL-C value was significantly increased in the metformin group $(P=0.023)$. GLP1 had an increasing trend in both groups, but it was only significant in the placebo group $(P=0.009)$. There were no significant changes in ALT, AST, hsCRP, FIAF and LPL concentrations during the study. 
Table 2 Effects of 2 months consumption of metformin and placebo on anthropometric indices and biochemical variables in obese women.

\begin{tabular}{|c|c|c|c|c|c|}
\hline \multirow[b]{2}{*}{ Variables* } & \multicolumn{2}{|c|}{ Metformin $(n=20)$} & \multicolumn{2}{|c|}{ Placebo $(n=16)$} & \multirow[b]{2}{*}{ P value** } \\
\hline & Baseline & After intervention & Baseline & After intervention & \\
\hline \multicolumn{6}{|l|}{ Body composition } \\
\hline Weight (kg) & $90.9 \pm 10.8$ & $86.9 \pm 11.2^{\dagger}$ & $86.8 \pm 6.3$ & $84.5 \pm 6.6^{\ddagger}$ & 0.06 \\
\hline BMI $\left(\mathrm{kg} / \mathrm{m}^{2}\right)$ & $35.0 \pm 3.4$ & $33.4 \pm 3.5^{\dagger}$ & $32.7 \pm 2.2$ & $31.8 \pm 2.6^{\ddagger}$ & 0.016 \\
\hline Waist (cm) & $102.8 \pm 7.3$ & $97.5 \pm 8.7^{\dagger}$ & $98.9 \pm 5.3$ & $94.9 \pm 5.0^{\ddagger}$ & 0.34 \\
\hline $\operatorname{Hip}(\mathrm{cm})$ & $121.3 \pm 7.3$ & $116.5 \pm 8.3^{\dagger}$ & $115.3 \pm 7.1$ & $112.2 \pm 7.0^{\dagger}$ & 0.08 \\
\hline WHR & $0.85 \pm 0.05$ & $0.84 \pm 0.04^{t \dagger}$ & $0.86 \pm 0.06$ & $0.85 \pm 0.05$ & 0.95 \\
\hline WHtR & $0.64 \pm 0.05$ & $0.60 \pm 0.05^{\dagger}$ & $0.61 \pm 0.02$ & $0.58 \pm 0.03^{\dagger}$ & 0.23 \\
\hline \multicolumn{6}{|l|}{ Glucose metabolism } \\
\hline FBS (mg/dL) & $81.0 \pm 9.13$ & $84.55 \pm 8.63$ & $80.81 \pm 11.93$ & $84.31 \pm 8.49^{t \dagger}$ & 0.60 \\
\hline Insulin $(\mathrm{mU} / \mathrm{L}) * \star \star$ & $15.78 \pm 25.76$ & $8.31 \pm 7.97^{t \dagger}$ & $26.53 \pm 54.92$ & $6.10 \pm 4.00$ & 0.39 \\
\hline HOMA-IR*** & $3.30 \pm 5.76$ & $1.77 \pm 1.77$ & $5.13 \pm 10.56$ & $2.15 \pm 3.65$ & 0.74 \\
\hline \multicolumn{6}{|l|}{ Lipid profile } \\
\hline $\mathrm{TC}(\mathrm{mg} / \mathrm{dL})$ & $173.95 \pm 38.26$ & $177.25 \pm 40.34$ & $183.12 \pm 35.65$ & $174.94 \pm 32.28^{t t}$ & 0.013 \\
\hline $\mathrm{TG}(\mathrm{mg} / \mathrm{dL})$ & $140.12 \pm 59.27$ & $135.15 \pm 42.80$ & $145.77 \pm 46.06$ & $162.87 \pm 73.87$ & 0.87 \\
\hline LDL (mg/dL) & $105.45 \pm 29.08$ & $112.95 \pm 34.57^{\dagger \dagger}$ & $115.25 \pm 28.84$ & $107.75 \pm 25.94^{\dagger \dagger}$ & 0.002 \\
\hline $\mathrm{HDL}(\mathrm{mg} / \mathrm{dL})$ & $39.75 \pm 10.90$ & $42.40 \pm 9.58^{\dagger \dagger}$ & $38.87 \pm 6.29$ & $40.37 \pm 5.61$ & 0.46 \\
\hline \multicolumn{6}{|l|}{ Liver markers } \\
\hline $\operatorname{ALT}(\mathrm{U} / \mathrm{L}) * * *$ & $13.62 \pm 7.36$ & $12.85 \pm 6.41$ & $11.36 \pm 4.66$ & $13.25 \pm 5.81$ & 0.33 \\
\hline AST $(U / L))^{\star \star *}$ & $22.00 \pm 8.92$ & $19.45 \pm 4.55$ & $18.95 \pm 3.99$ & $19.19 \pm 5.94$ & 0.96 \\
\hline \multicolumn{6}{|l|}{ Satiety hormone } \\
\hline 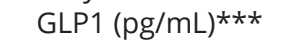 & $40.41 \pm 35.93$ & $97.28 \pm 120.88$ & $28.42 \pm 10.80$ & $164.36 \pm 143.66^{\ddagger}$ & 0.51 \\
\hline \multicolumn{6}{|l|}{ Inflammatory marker } \\
\hline $\mathrm{hsCRP}(\mathrm{mg} / \mathrm{L}) * \star *$ & $4.15 \pm 3.69$ & $7.89 \pm 16.73$ & $3.13 \pm 2.97$ & $5.08 \pm 5.11$ & 0.32 \\
\hline \multicolumn{6}{|l|}{ Adipose factors } \\
\hline FIAF (ng/mL) & $82.55 \pm 20.34$ & $78.35 \pm 25.82$ & $72.06 \pm 19.79$ & $69.18 \pm 15.72$ & 0.93 \\
\hline $\operatorname{LPL}(\mathrm{pg} / \mathrm{mL})^{\star \star *}$ & $362.06 \pm 142.88$ & $577.89 \pm 779.32$ & $392.51 \pm 131.12$ & $851.22 \pm 789.16$ & 0.12 \\
\hline $\begin{array}{l}\text { Significant difference with } \\
\text { *Data are presented as } \mathrm{m} \\
\text { and } \mathrm{BMI} ;{ }^{* *} \text { statistical tes } \\
\mathrm{ALT} \text {, alanine aminotransfe } \\
\text { GLP-1, glucagon-like pept } \\
\text { sensitive } \mathrm{C} \text { reactive protei } \\
\text { ratio; } \mathrm{WH} \mathrm{H} \text {, waist to heig }\end{array}$ & $\begin{array}{l}\text { roughout the study } \\
\text { comparisons betwe } \\
\text { d on log-transforme } \\
\text { spartate aminotran } \\
\text { high density lipoprot }\end{array}$ & $\begin{array}{l}0.001,{ }^{\ddagger} P<0.01,{ }^{\dagger \dagger} P \\
2 \text { groups were made } \\
\text { ta. } \\
\text { ase; BMI, body mass } \\
\text { cholesterol; HOMA- } \\
\text { esterol; LPL, lipopro }\end{array}$ & $\begin{array}{l}\text { red samples } t \text { test } \\
\text { aralized linear moc } \\
\text { S, fasting blood su }\end{array}$ & $\begin{array}{l}\text { ting for baseline me } \\
\text { fasting-induced adip } \\
\text { insulin resistance; }\end{array}$ & factor; \\
\hline
\end{tabular}

\section{Effect of intervention on microbial diversity, overall microbiota composition and enterotypes}

Comparable microbial diversity (Richness and Simpson indices) was observed in both groups before and after treatment $(P>0.05$, Fig. 2). Before metformin treatment, diversity indices correlated positively with LPL (Richness, $r=0.47, P=0.036$, Simpson, $r=0.58, P=0.007$ ). After the intervention, Simpson index was negatively correlated with BMI, waist, insulin and HOMA $(r=-0.48,-0.51$, -0.57 and -0.53 , respectively, $P<0.05)$. We visualized Bray-Curtis distances between samples using principle coordinate analysis (PCoA, Fig. 3). There was no separation based on the interventions, indicating that the overall gut microbiota was more dependent on interindividual variation rather than interventions.

Distribution of enterotypes, community clusters characterized by differences in the abundance of signature taxa, is shown in Fig. 4. Although Bacteroides 2 showed an increasing trend after metformin intake and the Bacteroides 1 enterotype increased after placebo intake in exchange for Bacteroides 2, these changes were not statistically significant $(P=0.85)$, and the distribution of enterotypes was not statistically different between before and after metformin intake $(P=0.77)$.

\section{Effect of intervention on specific fecal bacterial genus abundances}

Despite the absence of global signals, we did observe changes in the relative abundance of specific fecal bacterial genera after metformin intake (Fig. 5). For this analysis, we focused on the alteration of taxa whose concentration was changed after metformin treatment in previous studies $(12,15,31)$. Using this targeted analysis, we observed a significant increase in Escherichia/Shigella abundance (FDR-adjusted $P$ value $=0.012$ ). Moreover, 

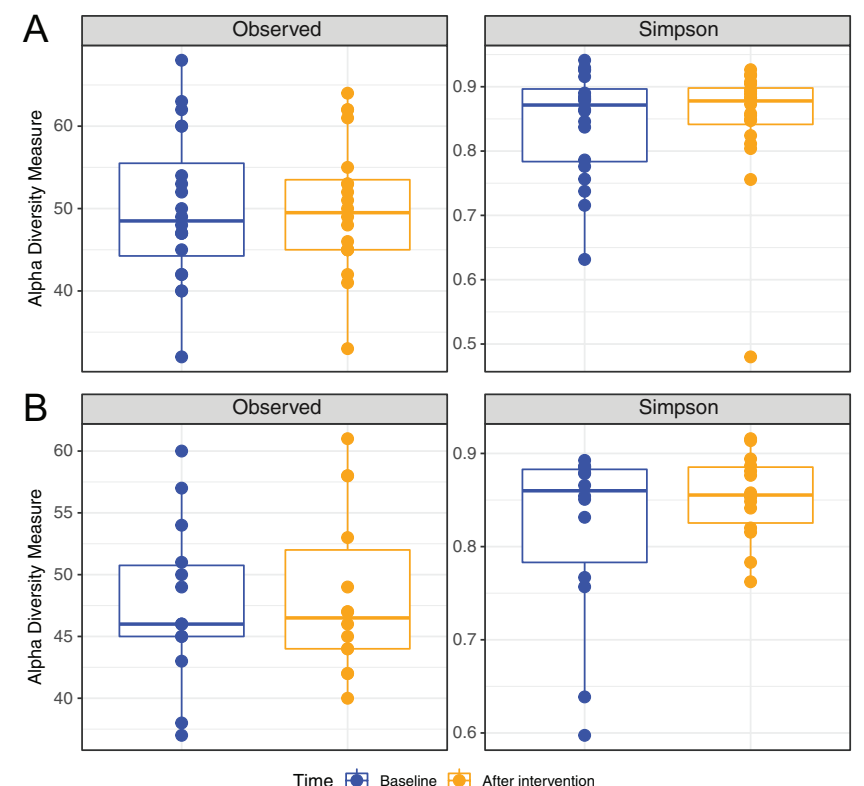

Time $\emptyset$ Baseline $\emptyset$ After intervention

\section{Figure 2}

Gut microbial diversity indices before and after (A) metformin $(n=20)$ or $(B)$ placebo $(n=16)$ treatment in obese women.

Color is indicative of time. A full colour version of this figure is available at https://doi.org/10.1530/EJE-18-0826.

a decrease in Intestinibacter abundance was observed, which did not remain significant following correction for multiple testing (FDR-adjusted $P$ value=0.19). The abundances of some of the SCFA-producing bacteria including Prevotella, Faecalibacterium, Lactobacillus and Akkermansia had mild non-significant decrease and some other ones including Roseburia, Blautia, Bacteroides and Butyrivibrio had mild non-significant increase in the metformin group. The abundance of Streptococcus had non-significant increasing trend in the metformin group. There was no significant change in relative abundances of bacterial genus in the placebo group. Escherichia/Shigella was also more abundant in the post-metformin group compared to the post-placebo group when adjusted for age and baseline BMI $(P=0.026)$.

The Prevotella-to-Bacteroides ratio remained unchanged in eight subjects and decreased in most of people (11 subjects) after metformin treatment. This ratio increased in five subjects after placebo + LCD and decreased or stayed unchanged in other participants of this group.

\section{Effect of intervention on fecal SCFAs}

Fecal SCFA concentrations were decreased in the metformin group during the study; however, they showed
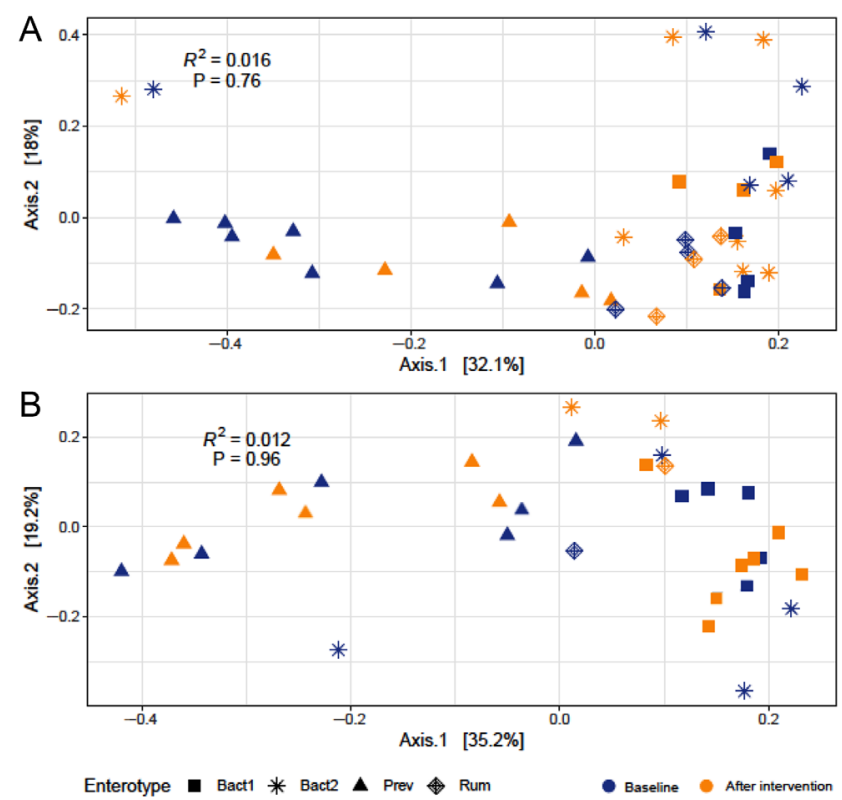

Figure 3

PCoA ordination of Bray-Curtis distances between samples before and after (A) metformin ( $n=20)$ or (B) placebo $(n=16)$ treatment in obese women. Each data point represents an individual sample. Symbol is indicative of enterotypes and color is indicative of time. A full colour version of this figure is available at https://doi.org/10.1530/EJE-18-0826.

an increasing trend in placebo (Fig. 6). Fecal concentration of acetate was significantly higher in the placebo group after intervention adjusted for baseline value and BMI $(P=0.002)$. Differences between the two groups in fecal propionate and butyrate levels were not statistically significant.

\section{Discussion}

In this 8-week randomized double-blind controlled clinical trial on obese women, we demonstrated that metformin $+\mathrm{LCD}$, compared with placebo $+\mathrm{LCD}$, results in a reduction of anthropometric indices, insulin concentration and fecal SCFA concentrations as well as an increased HDL-C without significantly changing the overall fecal microbial composition and diversity. We observed a significant specific increase in the Escherichia/ Shigella abundance after metformin intervention. We also found no significant changes in distribution of enterotypes, lipid metabolism, liver markers and satietyregulating hormone.

We found a significant reduction in weight, waist and hip circumferences in obese women who 
A

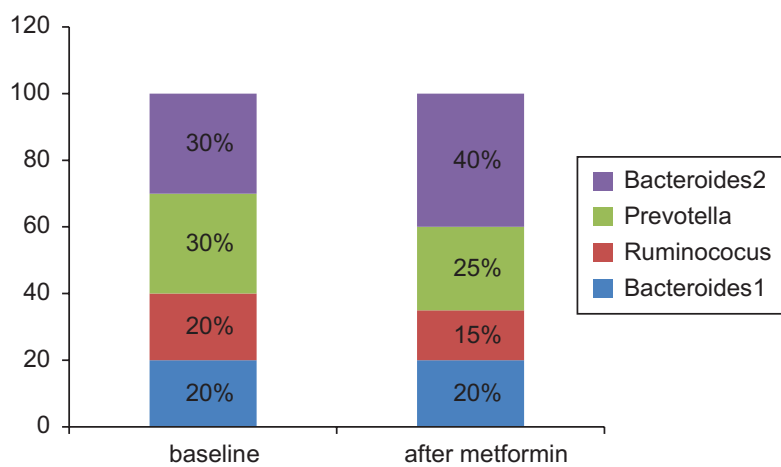

B

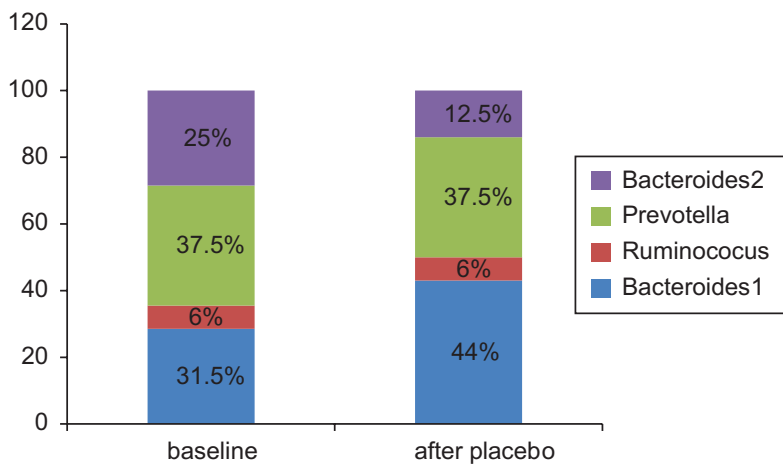

Figure 4

Distribution of the gut microbiome enterotypes before and after (A) metformin $(n=20)$ or (B) placebo $(n=16)$ treatment in obese women. Color is indicative of enterotypes. A full colour version of this figure is available at https://doi.org/10.1530/ EJE-18-0826.

were treated with metformin. A German multicenter analysis on 9108 diabetic patients showed that women had a significantly higher reduction of body weight compared to men after metformin treatment (32). There is growing evidence suggesting the weightreducing potential of metformin in nondiabetic obese subjects $(6,33)$. A systematic review indicated that orlistat and metformin had similar effects in reducing BMI of overweight/obese women with polycystic ovary syndrome (PCOS) (34).

In the present study, global gut microbial diversity indices were unaffected in the metformin group. However, in the metformin group, we identified negative correlations between Simpson Index and BMI, and waist and insulin resistance. A large study of American adults also showed that gut bacterial richness was lower in obese subjects compared to healthy-weight participants (35).

As our prescribed diet was mild, no notable effects on gut microbiota had been seen in the placebo group of this study, although previous restricted dietary interventions showed alterations in gut microbiota of obese individuals

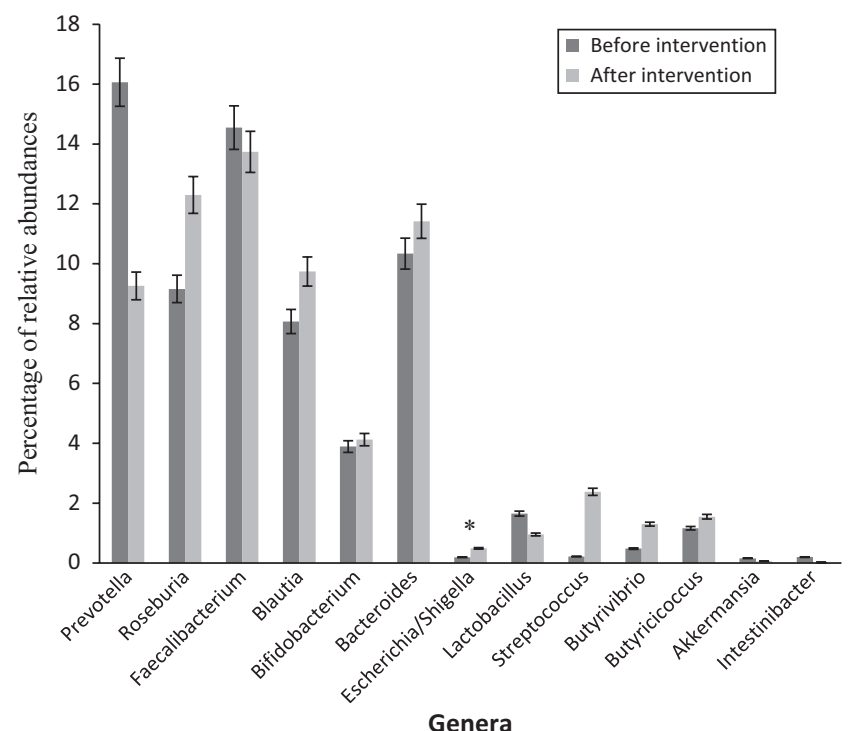

Figure 5

Changes in the relative abundance of fecal bacterial genus after metformin $(n=20)$ treatment in obese women. Color is indicative of time. ${ }^{*} P<0.05$.

$(36,37)$. Since the dietary recommendations were the same in both groups and no significant change in gut microbiota was observed in the placebo group, gut microbial alteration in the metformin group could be attributed to the metformin intervention.

In this clinical trial, we investigated the effects of metformin on the gut microbiota composition of nondiabetic obese adults, while prior studies were done on diabetic patients. Ma et al. (2018) showed that the microbiota profile of healthy mice treated with metformin had similarity with microbiota of prediabetes and irritable bowel syndrome situations (17). Intestinal discomforts which were prevalent side effects of metformin could be a consequence of relative increase in Escherichia abundance (38). We observed an increase of Escherichia abundance in the metformin-treated obese women. Escherichia is a gram-negative hydrogen producer bacterial genus contributing to hydrogen sulfide production (39). Despite the difference in the physiological conditions of target groups, we observed an increase in Escherichia abundance in the gut microbiota of metformin-treated obese women in agreement with previous observational studies on metformin-treated type 2 diabetic patients $(12,15,40$, 41); however, in vitro analysis suggested that the effect of metformin on Escherichia was indirect and might be results of other changes within the gut environment including reduced intestinal lipid absorption and LPS caused 
A

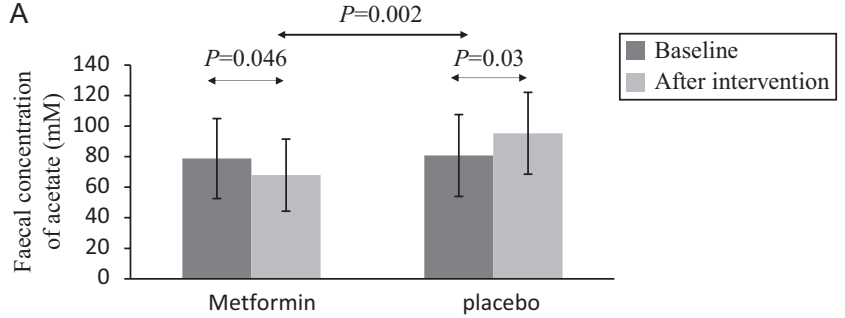

B

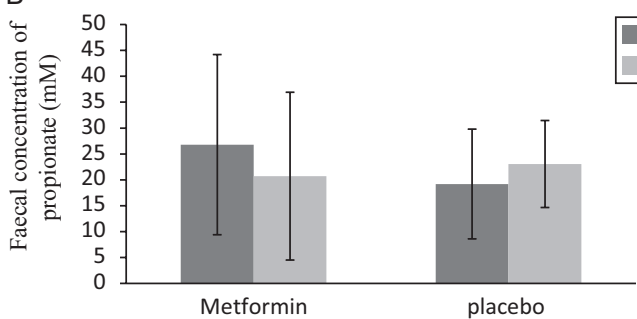

C

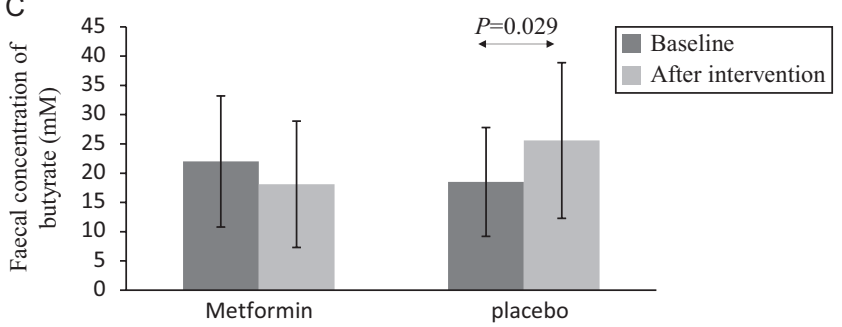

\section{Figure 6}

Concentration of fecal short chain fatty acids (SCFAs) before and after metformin $(n=20)$ or placebo $(n=16)$ treatment in obese women. Color is indicative of time. (A) Acetate, (B)

Propionate, (C) Butyrate.

inflammation $(31,42)$. Besides, metagenomic analysis reported that LPS biosynthesis by gram-negative bacteria was increased by metformin intervention $(15,42)$.

Million et al. (2013) in a study on 263 obese, overweight and lean individuals found for the first time that E. coli was negatively correlated with BMI (43). In alignment with this finding, we showed that BMI reduction associated with Escherichia increase in gut microbiota after metformin intake. As a probable underlying mechanism, Breton et al. (2016) in an animal study showed that meal-induced release of commensal Escherichia coli proteins into gut lumen could induce satiety and affect food intake. This $\alpha$-MSHlike protein, caseinolytic protease $\mathrm{B}(\mathrm{ClpB})$, activates gut enteroendocrine cells and stimulates secretion of the satietogenic hormones including glucagon-like peptide-1 (GLP-1) and peptide YY (PYY). ClpB also activates anorexigenic neurons in the brain, influencing shortterm and long-term dietary intakes (44). Confirming this hypothesis, an increasing trend observed in GLP-1 concentration after metformin intervention in this study could be a result of increased in Escherichia abundance and its satietogenic protein, although this increasing trend did not reach significance.

In obese rats, metformin induced enrichment of SCFA-producing bacteria, including Bacteriodes, Blautia and Butyricoccus, and reduction of microbial diversity (45). However, we identified only subtle and inconsistent changes in different SCFA-producing bacterial genus in the intervention group. We showed a non-significant reduction of Intestinibacter, a butyrate-producing bacterium, in the metformin-treated group confirming the findings of previous studies (12, 15). Akkermansia, a propionate producer, did not change significantly in the present study. Results of previous studies about Akkermansia were not very conclusive too $(11,15)$. Forslund et al. (2015) observed inconsistent trends of Akkermansia between different country subsamples after metformin intervention in type 2 diabetes (12). Contrary to our results, some previous studies declared an increase in Bifidobacterium of gut microbiome following metformin intake (15). Roseburia which has anti-inflammatory and anti-glycemic effects had a lower abundance in obese individuals compared to lean subjects (46). In this study, we showed that the amount of Roseburia had a nonsignificant increase during metformin intervention, making gut microbiota of obese subjects more similar to lean subjects. These differences in observed results about gut bacterial abundance could highlight the complexity of interactions between gut microbiota and interventions and might be explained by the inter-individual variation of microbiota between obesity and diabetic situations and differences of intestinal microbiota composition as a result of confounding factors like immune response, age, dietary intakes, ethnicity and geographical locations $(16,47)$. The lack of significant results other than Escherichia/Shigella could be linked to lack of power - the study was powered on clinical endpoints. Recent estimates suggest that several hundreds of individuals in each arm would be necessary toward this aim (29).

Previous studies candidate increased production of SCFAs as a potential mechanism for health benefits of metformin via gut microbiota (11). However, our study failed to show an increase in fecal SCFA concentrations after metformin intake. Wu et al. (2017) showed that metformin intervention for 4 months caused an increase in fecal propionate and butyrate concentrations in diabetic men; however, no differences were observed after combining men and women (15). We also observed that SCFA concentrations in obese women were reduced 
by metformin, pointing the importance of gender in microbiota manipulation. The gender-dependent effect of dietary interventions on gut microbiota has also been indicated in previous studies $(48,49)$.

The gut microbiota-regulated FIAF could impact on fat storage via inhibiting LPL activity (20). Previous animal studies have suggested that FIAF is possibly modulated by SCFAs (20); however, in this study, despite the reduced fecal SCFAs, no statistically significant differences existed in FIAF and LPL concentrations after metformin intervention.

Previous studies proposed that Prevotella-to-Bacteroides ratio in gut microbiota could predict the response of obese subjects to dietary interventions $(50,51)$. In the present study, we showed that this ratio remained unchanged or decreased after metformin +LCD treatment in obese women.

To the best of our knowledge, the effect of metformin on gut microbiota of healthy obese female has never been reported. By conducting the clinical trial and investigating the effect of metformin on paired samples, the effect of interindividual variations was reduced in this study in comparison with previous case-control studies. Moreover, previous cross-sectional studies could not determine causality, highlighting the need for clinical trials. Furthermore, our healthy participants had not used metformin before, nullifying the potential impact of different duration of therapy on the microbiota. By excluding subjects who had used antibiotics and weight loss drugs a few months prior to study, the effects of those two major confounding factors on gut microbiota were eliminated. Besides, lowcalorie diet was the same in the two groups, normalizing the effect of diet in any potential change in the gut microbiota. It should be noted that we cannot conclude if the weight-reducing effect of metformin was through the gut microbiota or other probable mechanisms, including appetite suppression, improvement of insulin sensitivity and regulation of fat oxidation and storage that should be considered. Additional studies combining metagenomics and untargeted metabolomics analyses are needed to clarify the effect of metformin on weight control through gut microbiota modulation. Shotgun sequencing should be applied to allow assessment of gut microbiota response at functional levels. Furthermore, due to the effective role of gender on microbiota, the effect of metformin on obese men should be investigated too. As one of the limitations of this trial is its sample size, large-scale interventional studies with higher power are needed to confirm the results.

\section{Conclusion}

In summary, we found that metformin supplementation in addition to low-calorie diet, compared to placebo supplemented low-calorie diet, resulted in BMI reduction and specific increased abundance of gut Escherichial Shigella in non-diabetic obese Iranian women. No statistically significant differences for overall microbiota composition were observed after multiple testing adjustments. To disentangle power issues vs true absence of signal, additional larger microbiome-endpoint powered studies are needed.

\section{Declaration of interest}

The authors declare that there is no conflict of interest that could be perceived as prejudicing the impartiality of this study.

\section{Funding}

This work was supported by the National Institute for Medical Research Development (NIMAD) (grant number: 942022).

\section{Acknowledgement}

The drugs were kindly provided by KoushanpharmedCo.

\section{References}

1 Andolfi C \& Fisichella PM. Epidemiology of obesity and associated comorbidities. Journal of Laparoendoscopic and Advanced Surgical Techniques 201828 919-924. (https://doi.org/10.1089/lap.2018.0380)

2 Jafari-Adli S, Jouyandeh Z, Qorbani M, Soroush A, Larijani B \& Hasani-Ranjbar S. Prevalence of obesity and overweight in adults and children in Iran; a systematic review. Journal of Diabetes and Metabolic Disorders 201413 121. (https://doi.org/10.1186/s40200-014-0121-2)

3 Finkelstein EA, Khavjou OA, Thompson H, Trogdon JG, Pan L, Sherry B \& Dietz W. Obesity and severe obesity forecasts through 2030. American Journal of Preventive Medicine 201242 563-570. (https://doi.org/10.1016/j.amepre.2011.10.026)

4 Piche ME, Poirier P, Lemieux I \& Despres JP. Overview of epidemiology and contribution of obesity and body fat distribution to cardiovascular disease: an update. Progress in Cardiovascular Diseases 201861 103-113. (https://doi.org/10.1016/j. pcad.2018.06.004)

5 Hensley RD. Primary care management of obesity: individualized treatment strategies. Nurse Practitioner 201843 41-48. (https://doi. org/10.1097/01.NPR.0000534941.27466.0b)

6 Seifarth C, Schehler B \& Schneider HJ. Effectiveness of metformin on weight loss in non-diabetic individuals with obesity. Experimental and Clinical Endocrinology and Diabetes 2013121 27-31. (https://doi. org/10.1055/s-0032-1327734)

7 Malin SK \& Kashyap SR. Effects of metformin on weight loss: potential mechanisms. Current Opinion in Endocrinology, Diabetes and Obesity 201421 323-329. (https://doi.org/10.1097/ MED.0000000000000095)

8 Maniar K, Moideen A, Bhattacharyya R \& Banerjee D. Metformin exerts anti-obesity effect via gut microbiome modulation in prediabetics: a hypothesis. Medical Hypotheses 2017 104 117-120. (https://doi.org/10.1016/j.mehy.2017.06.001) 
9 Rodriguez J, Hiel S \& Delzenne NM. Metformin: old friend, new ways of action-implication of the gut microbiome? Current Opinion in Clinical Nutrition and Metabolic Care 201821 294-301. (https://doi. org/10.1097/MCO.0000000000000468)

10 Rothschild D, Weissbrod O, Barkan E, Kurilshikov A, Korem T, Zeevi D, Costea PI, Godneva A, Kalka IN, Bar N et al. Environment dominates over host genetics in shaping human gut microbiota. Nature 2018555 210-215. (https://doi.org/10.1038/nature25973)

11 de la Cuesta-Zuluaga J, Mueller NT, Corrales-Agudelo V, VelasquezMejia EP, Carmona JA, Abad JM \& Escobar JS. Metformin is associated with higher relative abundance of mucin-degrading akkermansia muciniphila and several short-chain fatty acid-producing microbiota in the gut. Diabetes Care 201740 54-62. (https://doi.org/10.2337/ dc16-1324)

12 Forslund K, Hildebrand F, Nielsen T, Falony G, Le Chatelier E, Sunagawa S, Prifti E, Vieira-Silva S, Gudmundsdottir V, Pedersen HK et al. Disentangling type 2 diabetes and metformin treatment signatures in the human gut microbiota. Nature 2015528 262-266. (https://doi.org/10.1038/nature15766)

13 Mardinoglu A, Boren J \& Smith U. Confounding effects of metformin on the human gut microbiome in type 2 diabetes. Cell Metabolism 201623 10-12. (https://doi.org/10.1016/j.cmet.2015.12.012)

14 Tong X, Xu J, Lian F, Yu X, Zhao Y, Xu L, Zhang M, Zhao X, Shen J, Wu S et al. Structural alteration of gut microbiota during the amelioration of human type 2 diabetes with hyperlipidemia by metformin and a traditional chinese herbal formula: a multicenter, randomized, open label clinical trial. MBio 20189 e02392-e024017. (https://doi.org/10.1128/mBio.02392-17)

$15 \mathrm{Wu} \mathrm{H}$, Esteve E, Tremaroli V, Khan MT, Caesar R, Manneras-Holm L, Ståhlman M, Olsson LM, Serino M, Planas-Fèlix M et al. Metformin alters the gut microbiome of individuals with treatment-naive type 2 diabetes, contributing to the therapeutic effects of the drug. Nature Medicine 201723 850-858. (https://doi.org/10.1038/nm.4345)

16 Ejtahed HS, Hasani-Ranjbar S \& Larijani B. Human microbiome as an approach to personalized medicine. Alternative Therapies in Health and Medicine 201723 8-9.

17 Ma W, Chen J, Meng Y, Yang J, Cui Q \& Zhou Y. Metformin alters gut microbiota of healthy mice: implication for its potential role in gut microbiota homeostasis. Frontiers in Microbiology 201891336. (https://doi.org/10.3389/fmicb.2018.01336)

18 Beaumont M, Goodrich JK, Jackson MA, Yet I, Davenport ER, VieiraSilva S, Debelius J, Pallister T, Mangino M, Raes J et al. Heritable components of the human fecal microbiome are associated with visceral fat. Genome Biology 201617 189. (https://doi.org/10.1186/ s13059-016-1052-7)

19 Ejtahed HS, Angoorani P, Hasani-Ranjbar S, Siadat SD, Ghasemi N, Larijani B \& Soroush AR. Adaptation of human gut microbiota to bariatric surgeries in morbidly obese patients: a systematic review. Microbial Pathogenesis 2018116 13-21. (https://doi.org/10.1016/j. micpath.2017.12.074)

20 Ejtahed HS, Soroush AR, Angoorani P, Larijani B \& Hasani-Ranjbar S. Gut microbiota as a target in the pathogenesis of metabolic disorders: a new approach to novel therapeutic agents. Hormone and Metabolic Research 201648 349-358. (https://doi.org/10.1055/s-0042-107792)

21 Tseng $\mathrm{CH} \& \mathrm{Wu} \mathrm{CY}$. The gut microbiome in obesity. Journal of the Formosan Medical Association 2018 Epub. (https://doi.org/10.1016/j. jfma.2018.07.009)

22 Esfahani FH, Asghari G, Mirmiran P \& Azizi F. Reproducibility and relative validity of food group intake in a food frequency questionnaire developed for the Tehran Lipid and Glucose Study. Journal of Epidemiology 201020 150-158. (https://doi.org/10.2188/ jea.JE20090083)

23 Mirmiran P, Esfahani FH, Mehrabi Y, Hedayati M \& Azizi F. Reliability and relative validity of an FFQ for nutrients in the Tehran lipid and glucose study. Public Health Nutrition 201013 654-662. (https://doi. org/10.1017/S1368980009991698)
24 Tito RY, Cypers H, Joossens M, Varkas G, Van Praet L, Glorieus E, Van den Bosch F, De Vos M, Raes J \& Elewaut D. Brief report: dialister as a microbial marker of disease activity in spondyloarthritis. Arthritis and Rheumatology 201769 114-121. (https://doi.org/10.1002/art.39802)

25 Hildebrand F, Tadeo R, Voigt AY, Bork P \& Raes J. LotuS: an efficient and user-friendly OTU processing pipeline. Microbiome 2014230. (https://doi.org/10.1186/2049-2618-2-30)

26 Callahan BJ, McMurdie PJ, Rosen MJ, Han AW, Johnson AJ \& Holmes SP. DADA2: high-resolution sample inference from Illumina amplicon data. Nature Methods 201613 581-583. (https://doi. org/10.1038/nmeth.3869)

27 Wang Q, Garrity GM, Tiedje JM \& Cole JR. Naive Bayesian classifier for rapid assignment of rRNA sequences into the new bacterial taxonomy. Applied and Environmental Microbiology $2007 \mathbf{7 3}$ 5261-5267. (https://doi.org/10.1128/AEM.00062-07)

28 Holmes I, Harris K \& Quince C. Dirichlet multinomial mixtures: generative models for microbial metagenomics. PLoS ONE 20127 e30126. (https://doi.org/10.1371/journal.pone.0030126)

29 Falony G, Joossens M, Vieira-Silva S, Wang J, Darzi Y, Faust K, Kurilshikov A, Bonder MJ, Valles-Colomer M, Vandeputte D et al. Population-level analysis of gut microbiome variation. Science 2016 352 560-564. (https://doi.org/10.1126/science.aad3503)

30 Benjamini Y \& Hochberg Y. Controlling the false discovery rate: a practical and powerful approach to multiple testing. Journal of the Royal Statistical Society: Series B (Statistical Methodology) $1995 \mathbf{5 7}$ 289-300. (https://doi.org/10.2307/2346101)

31 Montandon SA \& Jornayvaz FR. Effects of antidiabetic drugs on gut microbiota composition. Genes 20178 e250. (https://doi. org/10.3390/genes8100250)

32 Schutt M, Zimmermann A, Hood R, Hummel M, Seufert J, Siegel E, Tytko A, Holl RW; DPV initiative; German BMBF Competence Network Diabetes Mellitus. Gender-specific effects of treatment with lifestyle, metformin or sulfonylurea on glycemic control and body weight: a German multicenter analysis on 9108 patients. Experimental and Clinical Endocrinology and Diabetes 2015123 622-626. (https:// doi.org/10.1055/s-0035-1559608)

33 Malin SK, Nightingale J, Choi SE, Chipkin SR \& Braun B. Metformin modifies the exercise training effects on risk factors for cardiovascular disease in impaired glucose tolerant adults. Obesity 201321 93-100. (https://doi.org/10.1002/oby.20235)

34 Graff SK, Mario FM, Ziegelmann P \& Spritzer PM. Effects of orlistat vs. metformin on weight loss-related clinical variables in women with PCOS: systematic review and meta-analysis. International Journal of Clinical Practice 201670 450-461. (https://doi.org/10.1111/ ijcp.12787)

35 Peters BA, Shapiro JA, Church TR, Miller G, Trinh-Shevrin C, Yuen E, Friedlander C, Hayes RB \& Ahn J. A taxonomic signature of obesity in a large study of American adults. Scientific Reports 201889749. (https://doi.org/10.1038/s41598-018-28126-1)

36 Dao MC, Everard A, Aron-Wisnewsky J, Sokolovska N, Prifti E, Verger EO, Kayser BD, Levenez F, Chilloux J, Hoyles L et al. Akkermansia muciniphila and improved metabolic health during a dietary intervention in obesity: relationship with gut microbiome richness and ecology. Gut 201665 426-436. (https://doi. org/10.1136/gutjnl-2014-308778)

37 Haro C, Garcia-Carpintero S, Rangel-Zuniga OA, Alcala-Diaz JF, Landa BB, Clemente JC, Pérez-Martínez P, López-Miranda J, PérezJiménez F \& Camargo A. Consumption of two healthy dietary patterns restored microbiota dysbiosis in obese patients with metabolic dysfunction. Molecular Nutrition and Food Research 201761. (https://doi.org/10.1002/mnfr.201700300)

38 Hoffmann IS, Roa M, Torrico F \& Cubeddu LX. Ondansetron and metformin-induced gastrointestinal side effects. American Journal of Therapeutics 200310 447-451. (https://doi.org/10.1097/00045391200311000-00012) 
39 Rosario D, Benfeitas R, Bidkhori G, Zhang C, Uhlen M, Shoaie S \& Mardinoglu A. Understanding the representative gut microbiota dysbiosis in metformin-treated type 2 diabetes patients using genome-scale metabolic modeling. Frontiers in Physiology 20189775. (https://doi.org/10.3389/fphys.2018.00775)

40 Karlsson FH, Tremaroli V, Nookaew I, Bergstrom G, Behre CJ, Fagerberg B, Nielsen J \& Bäckhed F. Gut metagenome in European women with normal, impaired and diabetic glucose control. Nature 2013498 99-103. (https://doi.org/10.1038/nature12198)

41 Zhernakova A, Kurilshikov A, Bonder MJ, Tigchelaar EF, Schirmer M, Vatanen T, Mujagic Z, Vila AV, Falony G, Vieira-Silva S et al. Population-based metagenomics analysis reveals markers for gut microbiome composition and diversity. Science 2016352 565-569. (https://doi.org/10.1126/science.aad3369)

42 Winter SE, Winter MG, Xavier MN, Thiennimitr P, Poon V, Keestra AM, Laughlin RC, Gomez G, Wu J, Lawhon SD et al. Host-derived nitrate boosts growth of $E$. coli in the inflamed gut. Science 2013339 708-711. (https://doi.org/10.1126/science.1232467)

43 Million M, Angelakis E, Maraninchi M, Henry M, Giorgi R, Valero R, Vialettes B \& Raoult D. Correlation between body mass index and gut concentrations of Lactobacillus reuteri, Bifidobacterium animalis, Methanobrevibacter smithii and Escherichia coli. International Journal of Obesity 201337 1460-1466. (https://doi.org/10.1038/ijo.2013.20)

44 Breton J, Tennoune N, Lucas N, Francois M, Legrand R, Jacquemot J, Goichon A, Guérin C, Peltier J, Pestel-Caron M et al. Gut commensal E. coli proteins activate host satiety pathways following nutrientinduced bacterial growth. Cell Metabolism 201623 324-334. (https:// doi.org/10.1016/j.cmet.2015.10.017)

45 Zhang X, Zhao Y, Xu J, Xue Z, Zhang M, Pang X, Zhang X \& Zhao L. Modulation of gut microbiota by berberine and metformin during the treatment of high-fat diet-induced obesity in rats. Scientific Reports 20155 14405. (https://doi.org/10.1038/srep14405)

46 Tamanai-Shacoori Z, Smida I, Bousarghin L, Loreal O, Meuric V, Fong SB, Bonnaure-Mallet M \& Jolivet-Gougeon A. Roseburia spp.: a marker of health? Future Microbiology 201712 157-170. (https://doi. org/10.2217/fmb-2016-0130)

47 Yatsunenko T, Rey FE, Manary MJ, Trehan I, Dominguez-Bello MG, Contreras M, Magris M, Hidalgo G, Baldassano RN, Anokhin AP et al. Human gut microbiome viewed across age and geography. Nature 2012486 222-227. (https://doi.org/10.1038/nature11053)

48 Dominianni C, Sinha R, Goedert JJ, Pei Z, Yang L, Hayes RB \& Ahn J. Sex, body mass index, and dietary fiber intake influence the human gut microbiome. PLoS ONE 201510 e0124599. (https://doi. org/10.1371/journal.pone.0124599)

49 Bolnick DI, Snowberg LK, Hirsch PE, Lauber CL, Org E, Parks B, Lusis AJ, Knight R, Caporaso JG \& Svanbäck R. Individual diet has sex-dependent effects on vertebrate gut microbiota. Nature Communications 20145 4500. (https://doi.org/10.1038/ ncomms5500)

50 Hjorth MF, Blaedel T, Bendtsen LQ, Lorenzen JK, Holm JB, Kiilerich P, Roager HM, Kristiansen K, Larsen LH \& Astrup A. Prevotella-toBacteroides ratio predicts body weight and fat loss success on 24-week diets varying in macronutrient composition and dietary fiber: results from a post-hoc analysis. International Journal of Obesity 2018 Epub. (https://doi.org/10.1038/s41366-018-0093-2)

51 Roager HM, Licht TR, Poulsen SK, Larsen TM \& Bahl MI. Microbial enterotypes, inferred by the prevotella-to-bacteroides ratio, remained stable during a 6-month randomized controlled diet intervention with the new nordic diet. Applied and Environmental Microbiology 201480 1142-1149. (https://doi.org/10.1128/AEM.03549-13)

Received 10 October 2018

Revised version received 8 November 2018

Accepted 10 December 2018 